\title{
1 Electrostatic sampling of trace DNA from clothing
}

2 Author Information

3

$4 \quad$ Martin Zieger $^{1}$ (corresponding author)

$5 \quad$ Martin.Zieger@irm.unibe.ch

$6 \quad$ Tel.: +41316313159

7

8 Priscille Merciani Defaux ${ }^{2}$

$9 \quad$ pmep@police.be.ch

10

11 Silvia Utz ${ }^{1}$

$12 \quad$ Silvia.Utz@irm.unibe.ch

13

$14{ }^{1}$ Institute of Forensic Medicine, Forensic Molecular Biology Dpt., University of Bern, Sulgenauweg 40,

153007 Bern, Switzerland

$16 \quad{ }^{2}$ Bern Cantonal Police, Nordring 30, 3001 Bern, Switzerland

17 
During acts of physical aggression, offenders frequently come into contact with clothes of the victim, thereby leaving traces of DNA-bearing biological material on the garments. Since tape-lifting and swabbing, the currently established methods for non-destructive trace DNA sampling from clothing, both have their shortcomings in collection efficiency and handling, we thought about a new collection method for these challenging samples.

Testing two readily available electrostatic devices for their potential to sample biological material from garments made of different fabrics, we found one of them, the electrostatic dust print lifter (DPL), to perform comparable to well-established sampling with wet cotton swabs. In simulated aggression scenarios, we had the same success rate for the establishment of single aggressor profiles, suitable for database submission, with both the DPL and wet swabbing. However, we lost a substantial amount of information with electrostatic sampling, since almost no mixed aggressorvictim profiles suitable for database entry could be established, compared to conventional swabbing. This study serves as a proof of principle for electrostatic DNA sampling from items of clothing. The technique still requires optimization before it might be used in real casework. But we are confident that in the future it could be an efficient and convenient contribution to the toolbox of forensic practitioners.

Keywords: Trace DNA, Electrostatic, Clothing, Sampling 
Many criminal offenses, especially against physical or sexual integrity, involve a physical contact of the offender with clothing items of the victim. Sampling of the tiny amount of touch DNA that might be left by the offender in such a case is challenging for different reasons: The biological material deposited might enter the cavities of the tissue and might therefore be less readily retrieved than from smooth surfaces such as glass or plastic [1-3]. Fingerprints that would allow specific focusing on a small distinct area for sampling are hard to visualize on clothes. To complicate things even further, the garment has usually been worn by the victim for a certain period of time, thus carrying a lot of biological material not originating from the offender [4]. In routine casework, trace DNA from larger areas on clothing is usually sampled either by swabbing or by the application of adhesive tapes. Higher efficiency of DNA retrieval from most tested fabrics has been demonstrated by some studies for tape-lifting compared to swabbing $[5,6]$. However, sampling of larger areas by tape-lifting is tedious, especially since an optimal retrieval of trace material is achieved only after applying the tape several times consecutively in the same spot [6]. Swabbing is faster than tape-lifting and swab heads are very convenient for down-stream processing in the lab, but as previously mentioned, the literature states that DNA collection from textiles by swabs is less efficient. Also, thorough swabbing of clothing often results in frayed swab heads. If we presume that offenders are only in short contact with the item of clothing of a victim, we might expect them to leave only a relatively small amount of DNA on the garment. Thereby the exact origin and composition of the DNA-bearing biological material remains still unclear $[7,8]$. However, in most cases we would expect more biological material on the garment and absorbed by it as originating from the wearer [4]. A lot of this unwanted wearer DNA is usually co-sampled with wet swabs. Therefore a method by which one could minimize cosampling of aggressor DNA and victim DNA would be desirable. One approach could be to sample only material adhering loosely to the surface of the garment. This condition is partly fulfilled by the tape-lifting approach, though with the above mentioned inconvenience and a certain limitation for the size of the area that might be sampled by one tape. Here we tested two different electrostatic 
devices, frequently employed for other forensic purposes, for their potential for superficial trace removal from clothes. Both methods employ a film that is applied to the sample area and then charged electrically. Loose particles will adhere to the electrostatic film and can be swabbed from it subsequently. One device, the electrostatic detection apparatus $\left(\operatorname{ESDA}^{\circledR}\right)$, usually used to reveal indented writing on documents, has just very recently been demonstrated suitable for DNA retrieval from paper [9]. The second device that we tested, the electrostatic dust print lifter (DPL), is normally used to visualize foot prints on dusty floors. Therefore the footprints are covered by a metalized foil. The foil is then charged by applying high voltage with low amperage. Dust particles stick to the charged foil through electrostatic adhesion.

In the first part of this study we compared the DNA collection efficiency of the ESDA ${ }^{\circledR}$ and the DPL to conventional wet swabbing. The DPL, that turned out to be more efficient for DNA sampling, was then tested for its potential to limit co-sampling of wearer DNA in an aggressor-victim scenario. 
2.1 Sampling unworn clothes for comparison of swab, DPL and ESDA ${ }^{\circledast}$

We chose three different fabrics for sampling (Fig.1a): a pair of jeans (PJ), a cotton sweater (CS) and a wide-meshed jersey made of $85 \%$ acrylic fiber / 15\% cotton (WM). Swabs were taken as negative controls from $9 \times 13 \mathrm{~cm}$ areas on washed and untouched clothes. The clothes were touched by three different persons at three different areas by pressing both hands on the item of clothing followed by an outward movement of the hands (Fig.1b). Donors were told not to wash hands prior to sampling. Between two different garments to touch, there was a minimum time interval of 30 minutes for the donors. Every garment has been touched three times by the same person at three different days. The clothes were washed between sampling days. Every area was subdivided in three areas with the dimensions $9 \times 13 \mathrm{~cm}$. Every one of these $9 \times 13 \mathrm{~cm}$ areas was sampled with a different technique (Fig.1c): a wet cotton swab (Cardboard evidence collection Kit, Prionics, Switzerland), the dust print lifter (DPL) ESP900 (SIRCHIE, North Carolina, USA) or the electrostatic detection apparatus ESDA ${ }^{\circledR}$ (Foster \& Freeman, UK). Every time the sampling order was altered, to equilibrate for potential uneven deposit. We therefore sampled every garment for every donor once in every position (A, B or C) with every technique, resulting in a total of 27 samples per sampling technique. For sampling with the $\mathrm{ESDA}^{\circledR}$, the garment was put on the ESDA ${ }^{\circledast}$ table and covered with the Mylar film made of boPET (biaxially-oriented polyethylene terephthalate). The touched area was marked on the backside of the film and then the corona wand was passed over it to induce electrostatic charging of the film. After charging, the film was removed carefully to avoid folding. For sampling with the DPL, the metalized lifting foil was cut to $9 \times 13 \mathrm{~cm}$ pieces. The touched area was covered with the metal foil and the foil was charged with the maximum voltage for $15 \mathrm{~s}$. The electrostatic ESDA ${ }^{\circledR}$ and DPL films were swabbed with wet cotton swabs directly after the electrostatic sampling. Negative controls for unused ESDA ${ }^{\circledR}$ and DPL films were analyzed in triplicate. 
103

104

105

Three persons (victims) were wearing a pair of jeans (different colors) and a cotton sweater for at least one day. They were vigorously touched by three different persons (aggressors) at the forearms and ankles (Fig.2a and b) for about 5 seconds, simulating one person who holds another one trying to free itself, therefore including friction. The areas for sampling were subsequently limited to $10 \times 15$ $\mathrm{cm}$. One side (e.g. left arm / left leg) was then sampled with a wet cotton swab (Fig.2c) and the other one (right arm /right leg) with the DPL as described above (Fig.2d). DPL foils were wet swabbed directly after the electrostatic sampling. The sampling was repeated two days later with the same victim-aggressor combination, changing the sampling side (e.g. now DPL left, swab right). The two following weeks the combinations of aggressor and victim were altered. In the end every aggressor touched every one of the victims two times, at trousers and sweater, resulting in a total of 36 samples taken by swab and 36 taken by DPL.

\subsection{Sample analysis}

Cotton swabs were extracted using the AutoMateExpress ${ }^{\mathrm{TM}}$ Extraction with the PrepFiler Express $^{\mathrm{TM}}$ protocol from Life Technologies, Massachusetts, USA. DNA quantification was done by Real-TimePCR (qPCR) using the Quantifiler ${ }^{\circledast}$ Human Kit from Life Technologies on a 7500 RT PCR System (Applied Biosystems ${ }^{\circledR}$, Massachusetts, USA). DNA profiles were established by multiplex-PCR using the AmpFISTR ${ }^{\circledR}$ NGM Select ${ }^{\mathrm{TM}}$ Kit from Life Technologies. We used the recommended standard amplification protocol with $25 \mu$ l reaction volume. For samples with a DNA concentration lower than $50 \mathrm{pg} / \mu \mathrm{l}$, we used the maximum volume of $10 \mu \mathrm{l}$ sample for amplification. For higher concentrated samples we used a volume corresponding to $0.5 \mathrm{ng}$ per reaction and filled to $25 \mu$ l with water. In accordance with our internal guidelines for routine casework, negative controls and all samples with a DNA concentration lower than $20 \mathrm{pg} / \mu \mathrm{l}$ were amplified with 32 cycles, all other samples with 30 cycles. Electrophoresis was performed on a 3130xl Genetic Analyzer (Applied Biosystems ${ }^{\circledR}$, 
127 Massachusetts, USA) with $3 \mathrm{kV}$ injection voltage and 10 seconds injection time on POP4 polymer.

128 Results were analyzed with Genemapper ${ }^{\circledR}$ ID-X Version 1.4 (Applied Biosystems ${ }^{\circledR}$, Massachusetts, USA) with $50 \mathrm{rfu}$ (relative fluorescent units) threshold.

132 For the comparison of Swab, DPL and ESDA ${ }^{\circledR}$ on fresh clothing we compared the quantities of DNA

133 that could be sampled with the different techniques. Because of the large differences in DNA quantities retrieved, we preferred a classification of the samples in three different concentration classes over less meaningful mean DNA quantities for the different techniques. We chose DNA concentration classes for the retrieved extraction of $<10 \mathrm{pg} / \mu \mathrm{l}, 10-20 \mathrm{pg} / \mu \mathrm{l}$ and $\geq 20 \mathrm{pg} / \mu \mathrm{l}$ based on our experience from daily case work. For the evaluation of DNA profiles we counted at how many loci we could detect both alleles of the DNA donor and compared mean values of loci between sampling techniques as well as how often we could detect all alleles of the donor on the sampled garment. For the second comparison between swab and DPL on worn clothing we applied the entry criteria of the Swiss DNA database for classification. A partial single person profile or main component can be registered if a minimum of 6 loci has been characterized. For mixtures the minimum number of

143 determined loci is 8 . Mixtures can be registered if the submitted alleles indicate not more than two

144 contributors. For single profiles, we only accepted unambiguous major components with good

145 heterozygote balance (>60\% peak height ratio). Mixtures were checked for continuous distribution 146 of signal intensity of victim and aggressor alleles. 
Figure 1 shows the experiment setup of the first part of the study. The efficiency of the different sampling methods was checked by the quantity of DNA that could be retrieved (Table 1). Therefore we set three different concentration classes for the DNA extractions. Whereas half of the samples (13 out of 27 ) collected by the DPL gave concentrations of $10 \mathrm{pg} / \mu$ l or more, the ESDA ${ }^{\circledR}$ collection method only yielded two samples with a high DNA concentration ( $\geq 20 \mathrm{pg} / \mu \mathrm{l})$. The DPL collection method performed almost as good as conventional swabbing (17 out of 27 samples with a concentration of more than $10 \mathrm{pg} / \mu \mathrm{l})$. The wide-meshed jersey made of $85 \%$ acrylic fiber and $15 \%$ cotton accounts for the major difference between the different garments tested. For this clothing item collection efficiency is high for swabbing, whereas almost no DNA could be sampled with the two electrostatic devices. The established donor DNA profiles fit the detected DNA concentrations. Out of the 16 loci in the AmpFISTR ${ }^{\circledR}$ NGM Select ${ }^{\mathrm{TM}}$ multiplex, an average of 15.5 loci over all 27 samples could be established with sampling by swabs. The DPL performed similarly well with an average of 14.3 loci. Considerably fewer loci (6.7) could be characterized with the ESDA ${ }^{\circledR}$ collection method. Only $19 \%$ of the samples collected with the ESDA ${ }^{\circledR}$ device yielded complete donor profiles, compared to $70 \%$ for DPL and $85 \%$ for wet swabbing. The results are summarized in Table 1 . Control samples taken from untouched clothing and from the unused Mylar and metalized foils were all negative for qPCR.

\subsection{Sampling worn clothes for simulated aggression}

Figure 2 depicts the procedure for the second part of the study. Again we measured DNA concentrations in the samples by qPCR (Table 2). As for the first part of the study, the wet cotton swab was more efficient in overall DNA retrieval. We checked at how many of the AmpFISTR ${ }^{\circledR}$ NGM Select ${ }^{\mathrm{TM}}$ loci we could detect the alleles of the aggressor. The numbers for both methods are 
173 comparable to each other. With the DPL collection method we were able to detect in average $75 \%$ of

174 the aggressor profile, with swabbing $89 \%$. We then checked how many of the samples would yield a

175 single aggressor profile suitable for entry into the Swiss DNA database. Most of the cases we treat

176 during our daily work do not involve a known suspect. Therefore a good quality profile, fulfilling the

177 entry criteria for the database is crucial in most cases if the established profile shall serve as an

178 investigative lead. With both collection methods 5 out of 36 traces (14\%) yielded a database suitable

179 single donor profile. We expected wet swabbing to retrieve more of the wearer DNA. Consistent with

180 this presumption we obtain considerably more aggressor-donor mixtures suitable for database entry,

181 but numbers for single victim profiles were comparable between the two methods. Contamination

182 with DNA from unknown individuals was an issue with both sampling techniques, since almost all

183 profiles showed additional signals that could be assigned neither to the aggressor nor to the victim. 
Within less than 20 years, trace DNA analysis has become a standard procedure in forensics [10-12].

In our lab about $80 \%$ of all samples are so called "contact traces", traces that do presumably not involve any body fluids. Those traces mostly contain very small amounts of DNA and can usually not be visualized prior to sampling. Despite the obvious importance of this type of samples for forensics, established methods for sample collection are scarce. For smooth, solid surfaces such as glass, wood or plastic, swabs are still the method of choice because sampling is quick and relatively cheap and down-stream processing of swab heads is convenient. For items of clothing, tape-lifting has gained in importance, but down-stream processing of tapes is less handy. Although a variety of other collection methods for trace DNA have been presented by researchers, including single particle analysis to avoid mixtures or collection by vacuum [13-15], swabs and lifting tapes remain the two widely established methods in forensic labs. However, published data on comparison between tapes and swabs is still scarce. Most of the available publications on the topic are either case studies or include very few samples [5,16-18]. To our knowledge there is only one bigger systematic study publically available [6]. Verdon et al. demonstrate Scenesafe $\mathrm{FAST}^{\mathrm{TM}}$ tapes to be more efficient than swabbing on cotton and on a polyester/cotton mixture. For cotton, their paper also demonstrates that the tape has to be applied in the exact same spot more than once to be more efficient than swabbing. Verdon and colleagues demonstrate that in their setup, the highest sampling efficiency is achieved if the tape is applied 16 times in the same spot [6]. For an area of $100 \times 150 \mathrm{~mm}$ (or $15.000 \mathrm{~mm}^{2}$ ) as we use it here, we need to apply one Scenesafe FAST ${ }^{\mathrm{TM}}$ tape $\left(19 \times 25 \mathrm{~mm}\right.$ or $\left.475 \mathrm{~mm}^{2}\right) 32$ times to cover the whole surface once. So, to achieve the best sampling according to Verdon et al., we would need to apply the tape 512 times on that $10 \times 15 \mathrm{~cm}$ area. Since the above mentioned study showed as well that sampling efficiency starts decreasing after around 32 applications of the same tape we would need 16 tapes to cover a $10 \times 15 \mathrm{~cm}$ region under ideal sampling conditions. So even though in theory one might get most DNA sampled with this setup, it is far from being convenient for real case scenarios, with larger sampling areas involved. For sampling of trace DNA from garments that have 
been touched through physical aggression, the sampled areas are usually rather large, since mostly no fingerprints can be visualized to focus sample collection to smaller contact zones. Even if we assume that applying the tape only 3 times in the same spot would be sufficient for performing consistently better than swabbing, we would still need 96 contacts between tape and tissue and 3 tapes per sample for a $10 \times 15 \mathrm{~cm}$ area. Since the tapes need to be pooled to finally obtain the maximum DNA amount concentrated in one sample, every additional tape that has to be handled bears also an additional risk of contamination. To summarize, we acknowledge the better performance of adhesive tapes on smaller tissue areas, but from the existing literature, it is not yet clear which one is the best method for larger sampling areas, also in terms of usability. For this reason and because of the proof of principle character of the present study, we considered it at this stage neither necessary nor very informative to include an additional comparison of electrostatic sampling to tape-lifting.

For textiles, we can expect a transfer of DNA in both directions: from the item of clothing to the collection tool and also vice versa. As already mentioned above, it has been demonstrated for tapelifting that the tape reaches equilibrium for transfer of DNA-bearing biological material between the sampled garment and the tape [6]. We can expect a similar effect for the transfer of trace DNA between the garment and a swab. The rationale behind the experiments presented here was the following: if the loose biological material from textiles could be transferred to a plastic or metal film being neither sticky nor absorbent - then a large area can be sampled with a single swab, concentrating almost all the available DNA bearing material on that one swab and thereby also minimizing transfer of sampled DNA from the swab back to the clothing item. We expected such a collection method also to co-sample less biological material from the wearer of the clothing item, because the superficial electrostatic charge application would probably preferentially sample particles of skin abrasions and less wearer components such as sweat absorbed by the tissue.

We demonstrate here the potential of electrostatic methods for the collection of biological material for DNA profiling from clothing. Our observations are in line with the recent work from Plaza et al. 
[9]. However, we conclude that for electrostatic sampling from textiles, the electrostatic dust print

238 lifter (DPL) seems to be the better choice compared to the ESDA ${ }^{\oplus}$ device. The DPL did not only outperform the ESDA ${ }^{\oplus}$ in terms of DNA collection, we also found it more convenient to use. It is a portable device that can easily be brought to the crime scene and might therefore also be used for sampling from fixed surfaces such as carpeted floors. Also the electrostatic foil used for the DPL was easier to handle for swabbing following electrostatic collection than the Mylar film used on the $\mathrm{ESDA}^{\circledR}$.

Comparing different types of fabric, it was striking that the electrostatic sampling did not work well on the wide-meshed acrylic fiber jersey. One explanation for this observation could be that synthetic fibers better retain electrostatic charges [19] and might therefore compete with the applied electrostatic film. The swab worked best on the acrylic fibers, what is in line with a study demonstrating greater DNA transfer efficiency of biological material from synthetic fibers [3].

To further evaluate the collection efficiency of the DPL on cotton, we simulated physical aggression with three aggressors and three victims. Every one of the aggressors was touching the clothes of every one of the victims at two different days, resulting in a total of 72 samples, half of them sampled by wet swabbing, the other half by DPL. Almost all samples showed additional alleles from persons not participating in the experiments, as expected from the literature [20]. Since we could not detect any profile from the negative controls, we consider a contamination from the films unlikely, even though they are not manufactured for DNA collection purpose.

It was our intention to find a convenient sampling method for larger areas on items of clothing that yields the maximum of aggressor DNA while co-sampling a minimum of wearer DNA from the victim. We can conclude that the DPL is efficient for the collection of biological material originating from the aggressor, since we were able to establish the same number of single aggressor profiles by DPL as by conventional sampling. Unfortunately, our results did not show a specific enrichment of aggressor DNA, since we were able to establish a comparable number of victim profiles with swabs and with 
262 the electrostatic DPL. Also, the overall collection efficiency of the DPL appears to be somewhat lower

263 than swabbing, as can already be concluded from the results in Table 1. Due to this lower collection

264 efficiency, we lose a substantial amount of information in the form of mixed aggressor-victim profiles

265 suitable for database searching. So, even though the DPL showed its potential for sampling aggressor

266 DNA from larger areas, it appears not yet efficient enough to replace conventional sampling methods

267 because of the substantial loss in valuable mixed profile information. The mentioned limitations

268 might be overcome by more research on the method. Sampling efficiency might possibly be

269 improved by longer application of the electrostatic charge or by higher charges. The efficiency of

270 electrostatic charging might also depend on the size of the sampled surface. We could also imagine

271 electrostatic sampling as useful for larger surfaces that should not be swabbed directly due to PCR

272 inhibitor uptake through swabbing.

273 
275 We would like to thank Monique Zimmermann, Christoph Schneider and Emma Stoisser for technical

276 assistance, Britta Stoop and Mirco Hecht for critical reading of the manuscript and all volunteers for

277 their willingness to participate in the simulated aggression experiments.

278 
1. Goray M, Mitchell RJ, van Oorschot RAH (2010) Investigation of secondary DNA transfer of skin cells under controlled test conditions. Legal Med-Tokyo 12 (3):117-120.

doi:10.1016/j.legalmed.2010.01.003

2. Goray M, Eken E, Mitchell RJ, van Oorschot RAH (2010) Secondary DNA transfer of biological substances under varying test conditions. Forensic Sci Int-Gen 4 (2):62-67. doi:DOI 10.1016/j.fsigen.2009.05.001

3. Verdon TJ, Mitchell RJ, van Oorschot RAH (2013) The influence of substrate on DNA transfer and extraction efficiency. Forensic Sci Int-Gen 7 (1):167-175. doi:10.1016/j.fsigen.2012.09.004

4. Stouder SL, Reubush KJ, Hobson DL, Smith JL (2001) Trace Evidence Scrapings: A Valuable Source of DNA? Forensic Science Communications 3 (4)

5. Hansson O, Finnebraaten M, Heitmann IK, Ramse M, Bouzga M (2009) Trace DNA collectionPerformance of minitape and three different swabs. Forensic Sci Int-Gen 2 (1):189-190.

doi:10.1016/j.fsigss.2009.08.098

6. Verdon TJ, Mitchell RJ, van Oorschot RA (2014) Evaluation of tapelifting as a collection method for touch DNA. Forensic Sci Int-Gen 8 (1):179-186. doi:10.1016/j.fsigen.2013.09.005

7. Quinones I, Daniel B (2012) Cell free DNA as a component of forensic evidence recovered from touched surfaces. Forensic Sci Int-Gen 6 (1):26-30. doi:10.1016/j.fsigen.2011.01.004

8. Meakin G, Jamieson A (2013) DNA transfer: Review and implications for casework. Forensic Sci IntGen 7 (4):434-443. doi:10.1016/j.fsigen.2013.03.013

9. Plaza DT, Mealy JL, Lane JN, Parsons MN, Bathrick AS, Slack DP (2015) ESDA(R)-Lite collection of DNA from latent fingerprints on documents. Forensic Sci Int-Gen 16:8-12.

doi:10.1016/j.fsigen.2014.11.011

10. vanOorschot RAH, Jones MK (1997) DNA fingerprints from fingerprints. Nature 387 (6635):767767. doi:10.1038/42838

11. van Oorschot RA, Ballantyne KN, Mitchell RJ (2010) Forensic trace DNA: a review. Investigative genetics 1 (1):14. doi:10.1186/2041-2223-1-14

12. Wickenheiser RA (2002) Trace DNA: A review, discussion of theory, and application of the transfer of trace quantities of DNA through skin contact. Journal of forensic sciences 47 (3):442-450

13. Shalhoub R, Quinones I, Ames C, Multaney B, Curtis S, Seeboruth H, Moore S, Daniel B (2008) The recovery of latent fingermarks and DNA using a silicone-based casting material. Forensic science international 178 (2-3):199-203. doi:10.1016/j.forsciint.2008.04.001

14. Jiang X (2009) One method of collecting fallen off epithelial cell. Forensic Sci Int-Gen 2 (1):193. doi:10.1016/j.fsigss.2009.09.027

15. Farash K, Hanson EK, Ballantyne J (2015) Enhanced genetic analysis of single human bioparticles recovered by simplified micromanipulation from forensic 'touch DNA' evidence. Journal of visualized experiments : JoVE (97). doi:10.3791/52612

16. Bright JA, Petricevic SF (2004) Recovery of trace DNA and its application to DNA profiling of shoe insoles. Forensic science international 145 (1):7-12. doi:10.1016/j.forsciint.2004.03.016 17. May R, Thomson J (2009) Optimisation of cellular DNA recovery from tape-lifts. Forensic Sci IntGen 2 (1):191-192. doi:10.1016/j.fsigss.2009.08.115

18. Barash M, Reshef A, Brauner P (2010) The use of adhesive tape for recovery of DNA from crime scene items. Journal of forensic sciences 55 (4):1058-1064. doi:10.1111/j.1556-4029.2010.01416.x 19. Zilinskas PJ, Lozovski T, Jankauskas V, Jurksus J (2013) Electrostatic Properties and Characterization of Textile Materials Affected by Ion Flux. Mater Sci-Medzg 19 (1):61-66. doi:10.5755/j01.ms.19.1.3828

20. Goray M, van Oorschot RA (2015) The complexities of DNA transfer during a social setting. Legal Med-Tokyo 17 (2):82-91. doi:10.1016/j.legalmed.2014.10.003 

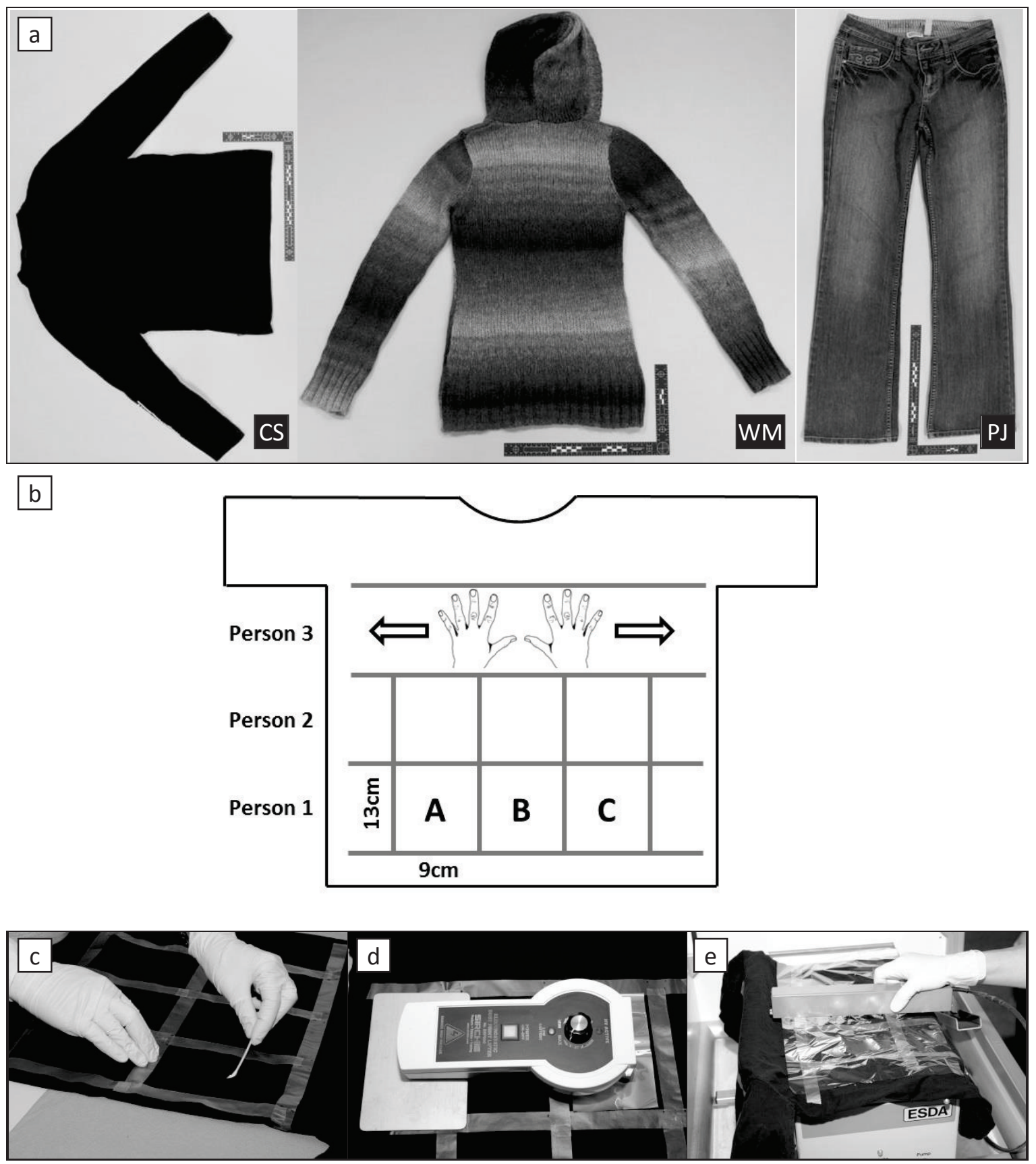

Figure 1 Experiment setup for the comparison of ESDA ${ }^{\circledR}$ and DPL DNA collection efficiency with wet cotton swabs. a) Three different garments were used for sampling. $C S=$ cotton sweater, $W M=$ wide-meshed jersey made of $85 \%$ acrylic fiber and $15 \%$ cotton, $\mathrm{PJ}=$ pair of jeans. b) Schematic illustration of the sample preparation. In a series of three experiments, every sampling technique has been used once in position A, B and C. c) Sampling by wet cotton swab. d) Sampling with the electrostatic dust print lifter (DPL). The metal foil is swabbed after charging. e) Sampling with the ESDA ${ }^{\circledR}$ device. The Mylar film is swabbed after charging. 


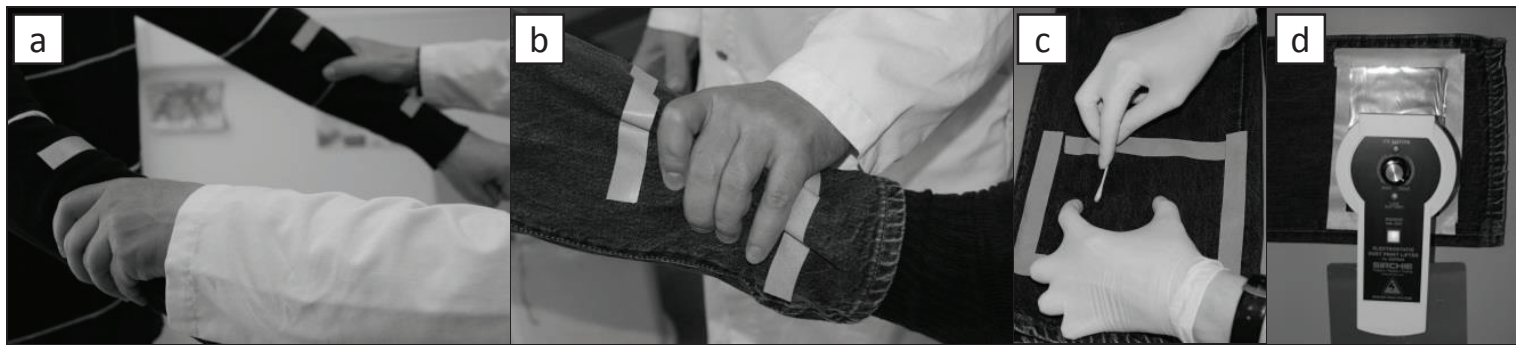

Figure 2 Simulated aggression. Victims carrying a cotton sweater and a pair of jeans were thoroughly touched at their a) forearms and $\mathbf{b}$ ) ankles. The areas were subsequently limited to $10 \times 15 \mathrm{~cm}$ and sampled either by c) wet cotton swab or d) 337 electrostatic dust print lifter (DPL). 
Table 1 Evaluation of collection efficiency of the ESDA ${ }^{\circledR}$ and DPL compared to conventional wet swabbing. DNA extractions were attributed to one of three concentration classes. Mean $\mathrm{N}^{\circ}$ of loci is the mean number of loci for which the donor alleles could be detected as major component with good heterozygote balance (>60\%). Complete profiles are profiles for which all 16 loci could be characterized with good heterozygote balance. $\mathrm{PJ}=$ pair of jeans, CS = cotton sweater, WM = wide-meshed jersey made of $85 \%$ acrylic and $15 \%$ cotton. SD = standard deviation.

\begin{tabular}{|c|c|c|c|c|c|c|c|c|c|c|c|c|}
\hline & \multicolumn{4}{|c|}{ Wet Swab $(n=27)$} & \multicolumn{4}{|c|}{ Sirchie DPL $(n=27)$} & \multicolumn{4}{|c|}{$\operatorname{ESDA}(n=27)$} \\
\hline & PJ & CS & WM & total & PJ & $\mathrm{CS}$ & WM & total & PJ & $\mathrm{CS}$ & WM & total \\
\hline$<10 \mathrm{pg} / \mu \mathrm{l}$ & 5 & 3 & 2 & $10(37 \%)$ & 4 & 2 & 8 & $14(52 \%)$ & 9 & 7 & 9 & 25 (93\%) \\
\hline $10-20 \mathrm{pg} / \mu \mathrm{l}$ & 2 & 2 & - & $4(15 \%)$ & 3 & 5 & - & $8(30 \%)$ & - & - & - & $0(0 \%)$ \\
\hline$\geq 20 \mathrm{pg} / \mu \mathrm{l}$ & 2 & 4 & 7 & $13(48 \%)$ & 2 & 2 & 1 & $5(19 \%)$ & - & 2 & - & $2(7 \%)$ \\
\hline Mean $N^{\circ}$ of Loci & \multicolumn{4}{|c|}{$15.5(S D \pm 1.4)$} & \multicolumn{4}{|c|}{$14.3(S D \pm 3.6)$} & \multicolumn{4}{|c|}{$6.7(S D \pm 6.3)$} \\
\hline Complete profiles & \multicolumn{4}{|c|}{$23(85 \%)$} & \multicolumn{4}{|c|}{$19(70 \%)$} & \multicolumn{4}{|c|}{$5(19 \%)$} \\
\hline
\end{tabular}


Table 2 Results from simulated aggression experiments. DNA concentration from every sample has been attributed to one of the three chosen concentration classes. Profiles have been evaluated for completely characterized loci. The entry criteria for the Swiss DNA database were applied. "Major single victim profile" means a profile that is not a mixture suitable for database (DB) submission but shows a clear major component originating from the victim. SD = standard deviation.

\begin{tabular}{c|c|c} 
& Wet Swab $(\mathrm{n}=36)$ & Sirchie DPL $(\mathrm{n}=36)$ \\
\hline$<10 \mathrm{pg} / \mu \mathrm{l}$ & $6 \%(2)$ & $58 \%(21)$ \\
$10-20 \mathrm{pg} / \mu \mathrm{l}$ & $17 \%(6)$ & $25 \%(9)$ \\
$>20 \mathrm{pg} / \mu \mathrm{l}$ & $78 \% \quad(28)$ & $17 \%(6)$ \\
\hline $\mathrm{N}^{\circ}$ Loci with aggressor alleles & $14.3(\mathrm{SD} \pm 3.0)$ & $12.0(\mathrm{SD} \pm 4.8)$ \\
DB suitable aggressor profiles $(\geq 6$ loci) & $14 \%$ & $14 \%$ \\
DB suitable mixtures $(\geq 8$ loci) & $36 \%$ & $6 \%$ \\
Major single victim profile $(\geq 6$ loci) & $25 \%$ & $22 \%$ \\
Profiles from more than 2 contributors & $100 \%$ & $92 \%$
\end{tabular}

